Jordà Bou, Damià.

Alumno de Doctorado en Artes: Producción e Investigación. Universitat Politécnica de València.

\author{
Sanmartín Piquer, Francisco Javier. \\ Profesor contratado doctor de la Universitat Politècnica de València.
}

\title{
Cultura audiovisual e imaginarios colectivos: El caso de las drogas en España.
}

\author{
TIPO DE TRABAJO
}

Comunicación.

PALABRAS CLAVE

Lenguajes audiovisuales, cultura social, drogas, reducción de riesgos.

KEY WORDS

Audiovisual languages, social culture, drugs, harm reduction.

\section{RESUMEN}

Esta comunicación se plantea como un estudio teórico dentro de la línea de investigación que relaciona lenguajes audiovisuales y cultura social. El principal objetivo es elaborar un análisis de las representaciones sociales del fenómeno de las drogas en España, a través de la máxima variedad de lenguajes, para describir la evolución de sus códigos de representación, la variedad de sujetos temáticos empleados y su importancia en la construcción del imaginario colectivo asociado a las drogas. Constituye una síntesis de la Tesis Doctoral que el autor ha realizado en este sentido, y se plantea como un extracto adaptado de tres casos de estudio concretos desde los que entender la metodología utilizada y, en cierta medida, algunas de las cuestiones observadas y las conclusiones alcanzadas.

\section{ABSTRACT}

This communication is intended as a theoretical study within the research that relates audiovisual languages and social culture. The main objective is to develop an analysis of the social representations of the drug phenomenon in Spain, through the widest range of languages, to describe the evolution of their codes of representation, the variety of thematic subjects used and their importance in building the collective imagination associated with drugs. It is a summary of the doctoral thesis that the author has made in this regard, and is presented as an excerpt from three specific case studies, in order to understand the methodology used and, to some extent, some of the observed issues and the conclusions reached.

\section{CONTENIDO}

Introducción

La forma en que entendemos las drogas no puede sino basarse en dos factores fundamentales. El primero es, obviamente, nuestra experiencia personal con ellas, bien sea directa o indirecta. Pero la segunda, quizá la que alberga más complicaciones y se nos escapa al control, es la percepción social y las diferentes representaciones que percibimos de este fenómeno, lo hagamos de forma consciente o inconsciente. Estas representaciones sociales cambian y oscilan con los tiempos, de forma que lo que en tiempos pudo estar muy bien visto, luego pasa a estar censurado, o viceversa. Las oscilaciones en el discurso social responden a varios factores 
sociológicos, políticos y económicos; y la mejor forma de visualizar estas conexiones en las últimas décadas es a través de sus formas de representación en los medios de comunicación de masas.

La percepción social que se vincula al consumo de substancias alteradoras de la conciencia, es determinada en base a ciertas formas de representación de este hecho cotidiano, desde los tiempos del relato mítico, hasta los mass media. Podemos decir por tanto que el fenómeno de la droga, y la percepción social que en cada momento histórico se tiene de él, viene indefectiblemente ligada a sus códigos de representación. Esta distorsión proyectada de la realidad, esas sombras que como en el mito de la caverna, se perciben en lugar del propio sujeto, constituyen uno de los más importantes activos en lo que se ha venido a denominar la construcción social del problema de la droga.

Eusebio Megías, actual director técnico de la Fundación de Ayuda contra la Drogadicción (FAD), define el concepto de representación social como "el conjunto sistemático de valores, nociones y creencias que permiten a los sujetos comunicarse y actuar, y así orientarse en el contexto social donde viven, racionalizar sus acciones, explicar eventos relevantes y defender su identidad" (Megías, 2001). Teniendo en cuenta esto, podemos afirmar que el problema de la droga es una representación social construida desde los medios de comunicación, en la que el interés de solucionar el problema, o como mínimo, de aportar una visión completa, heterogénea y socialmente transformadora, no ha sido nunca una prioridad.

El objetivo de esta comunicación es mostrar de forma breve y sencilla la metodología que, pretendiendo ahondar en los referentes que a lo largo de las últimas décadas han construido ese imaginario colectivo de las drogas a través de los lenguajes audiovisuales, ha constituido el cuerpo central de la Tesis Doctoral titulada "Vaselina en la lente. Las drogas en el imaginario audiovisual español (1960-2010)". Para ello, vamos a extraer tres casos de estudio que nos permitan entender esta metodología comparada utilizada en esta investigación, y que nos aporten una visión heterogénea al mismo tiempo que concreta y sintética.

\section{La Felicidad es la ausencia del dolor}

Caso 1- Publicidad: Calmante Vitaminado (1960)

El inicio de las emisiones de TVE da origen a un fenómeno que hoy entendemos como habitual, pero que en su momento constituyó una importante novedad: la publicidad audiovisual. En aquellos inicios del medio, sorprende la habitual utilización de técnicas de animación gráfica en los spots, generalmente de la mano de los prestigiosos Estudios Moro -aquellos que popularizaron a la Familia Telerín-. Destaca entre los primeros anuncios la abundancia de material relativo a fármacos que por entonces se publicitaba, casi siempre mediante los dibujos animados, algo que contrasta con la apariencia de seriedad científica que actualmente se acostumbra a emplear para incitarnos a comprar este tipo de productos. El popular Calmante Vitaminado, a base de ácido acetilsalicílico, que no era más que el primo ibérico de la internacional Aspirina de Bayer, era comercializado en nuestro país por la empresa Pérez Giménez. El principal ariete de la propaganda del producto se centraba en un concepto tan clásico -en el sentido más literal de la palabra- como la lucha contra el dolor. Los publicistas idearon una campaña protagonizada por un maestro helenístico que afirmaba categóricamente ante su alumno que "la felicidad es la ausencia del dolor". Este recurso coincide en gran medida con la representación antigua de Asclepio, dios de la medicina, que según cuenta una de sus leyendas, fue fulminado por Zeus porque osó revelar a los mortales el secreto de las virtudes del opio (o adormidera), hasta entonces privilegio de los Olímpicos (Escohotado, 1989).

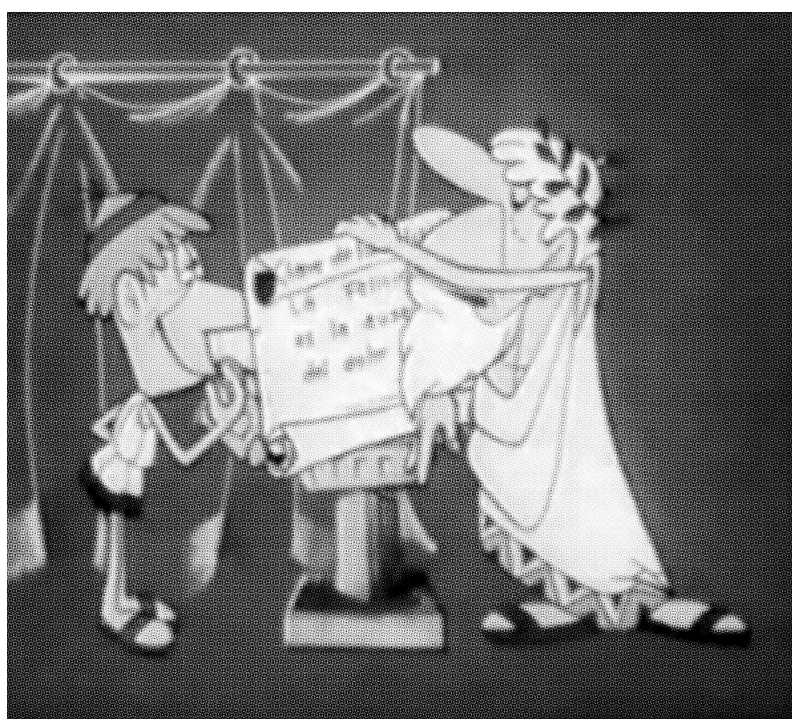


La relación que este spot establece entre el analgésico y los mitos históricos en torno al opio, puede resultar llamativa hoy en día. Sin embargo, en este referente radica una cuestión que se nos antoja mucho más reveladora: Asumir que se puede combatir el dolor fácilmente con un producto moderno rompe con el tabú moral cristiano de que el dolor es un castigo que los pecadores deben soportar. Esto establece un primer cambio de paradigma, en lo que respecta a los modos de consumo relativos a los fármacos, y que sentará las bases para la futura construcción social del problema de la droga. Esta campaña de Calmante Vitaminado ejemplifica a la perfección la proliferación de una cultura de intolerancia al dolor, es la síntesis de la tendencia a la curación tecnológica con fármacos, que hasta el momento no estaba en absoluto arraigada. Es especialmente rompedor si tenemos en cuenta que, en el momento histórico al que nos referimos, el colegio de médicos imponía serias restricciones a la hora de dispensar morfina por el hecho de considerarla adictiva. Además, existía el trasfondo moral de que el sacrificio tenía un valor, y estas prácticas farmacológicas transgreden el dogma de que es necesario asumir el dolor. Se trata del nacimiento en España de la llamada ideología biomédica, que pretende popularizar la idea de que la tecnología, en este caso farmacológica, es el medio más eficaz para solucionar nuestros problemas. Esta ideología biomédica es, no obstante, muy coherente con el Status Quo, pues en el fondo defiende que cualquier dolor -en el sentido más amplio de la palabra-, puede resolverse rápidamente con la aplicación de fármacos. Esto permite obviar todos los aspectos socio-culturales del dolor, del problema que nos atañe, en lo físico o en lo psicológico. Una ideología que en nuestros días es altamente dominante.

\section{Destape, Quinquis, Drogas, Morbo}

\section{Caso 2: Cine // Juventud Drogada // 1977}

La muerte de Franco trae como consecuencia en el cine un fenómeno bien conocido, el del "Destape". El cine más popular celebra la permisividad de la desnudez en pantalla, y lo hace de una forma que en ocasiones roza el esperpento. Esto deriva en la proliferación masiva de un cine que el mundo anglosajón llamaría de "serie B", en el que se busca el morbo con guiones pobres y bajos presupuestos, recurriendo para ello a temáticas sórdidas que empiezan en el erotismo pero terminan en la violencia, la delincuencia y, como no, la droga. La finalidad de toda esta cantidad de producciones cinematográficas es la rentabilidad comercial, al menos en su mayoría; pero tanto por su temática como por su exigua producción, con el tiempo irán ubicándose en categorías más bien undergound.

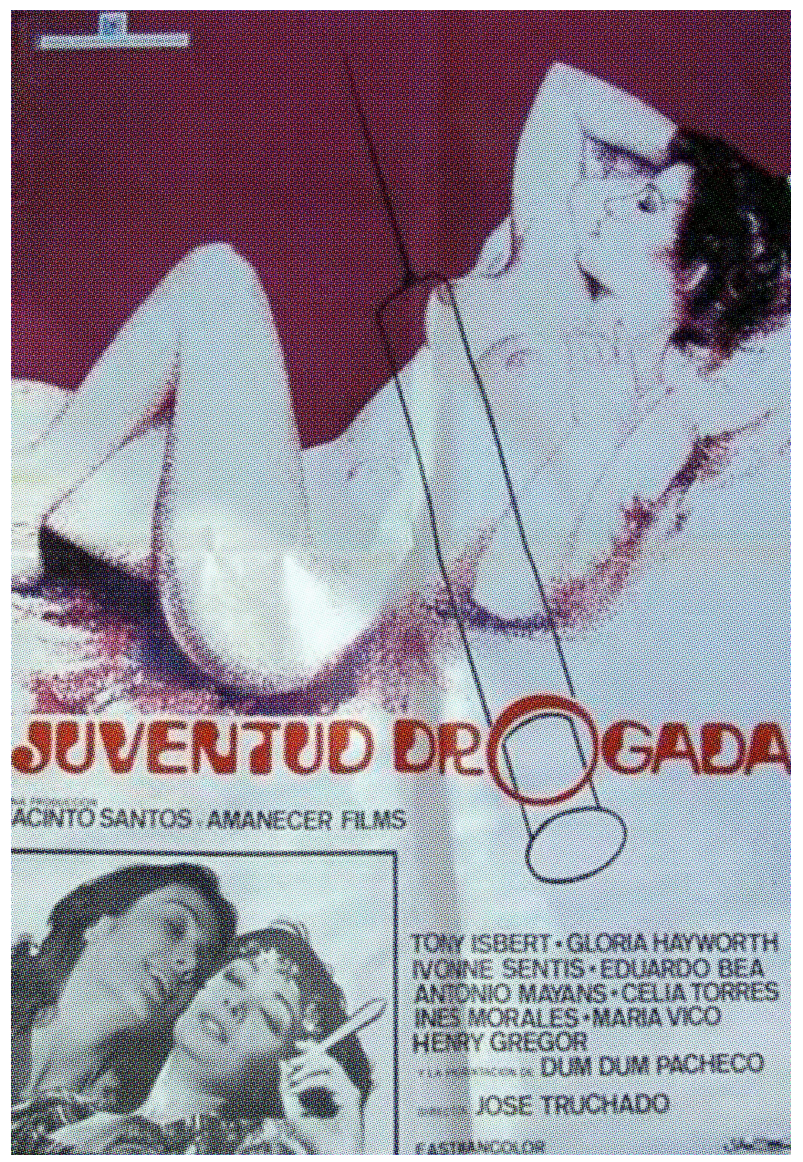


En este contexto aparece ese subgénero que se ha venido a denominar Cine Quinqui ${ }^{1}$, que contribuyó a fomentar la sensación de inseguridad y alarma social entre las clases medias y la sociedad "bien pensante", pero que al mismo tiempo estimulo la mitificación de determinadas conductas entre algunos sectores de la juventud trabajadora y pequeño burguesa. Estas películas pretendían retratar la realidad de los barrios periféricos de las grandes ciudades, -en los que proliferaba la delincuencia juvenil-, a través de unos relatos que, sin renunciar al dinamismo y la espectacularidad comercial, se adentraban casi de forma militante en la realidad del sujeto que retrataban, valiéndose de recursos tan sorprendentes como utilizar a los propios delincuentes como actores protagonistas -que para ello necesitaban en ocasiones de un permiso penitenciario-. El verdadero punto de inflexión para la creación del icono cultural quinqui llega en 1977, año en el que se certifica la primera muerte provocada por la heroína, y en el que el director José Antonio De La Loma estrena la exitosa cinta Perros Callejeros.

De La Loma fue el más representativo director de este subgénero, pero no fue el único. El mismo 1977 -año clave en la construcción social del problema de la droga- se estrenará otro film con un título premeditadamente escandalizador: Juventud Drogada, de José Truchado. Esta cinta -un tanto olvidada por la historia-, no se acerca tanto al subgénero quinqui, sino más bien a la pura explotation. En este caso el director dirige su objetivo hacia los jóvenes acomodados, alejándose del icono marginal, y toda la película esta envuelta de una atmósfera de excesos sexuales, pechos a raudales, crimen organizado, violencia y espectaculares jeringuillas (convertidas en el elemento icónico representativo del concepto "droga"). Este tipo de filmes que buscaban la provocación al amparo del destape y de la droga, sin más intención que el escándalo y sin ningún trasfondo moralizante -más que la obligada estigmatización interesada de algunas prácticas-, apenas conseguían provocar la reacción esperada en un segmento de público reducido, que difícilmente se veía identificado en esas pintorescas representaciones. Lo cierto es que pese al manido mensaje de "se empieza con el porro y te acabas convirtiendo en un adicto a la heroína que abandona a su familia", la cinta es sorprendente por la poca vergüenza y la ligereza con la que mezcla conceptos. Fiestas psicodélicas, gimnasios de matones y karatekas, comunas hippies y una triple felación forzada precedida por primeros planos de los paquetes del trío de violadores, son algunas de las lindezas que nos regala. Es en definitiva un revelador ejemplo de ese cine de "serie B", más dirigido a los amantes del terror y la acción (y el sexo, no lo olvidemos), que se servían de los absurdos tópicos de la droga para tejer inverosímiles tramas, y que poco o nada representaban la realidad social del fenómeno.

\section{Yonquis en Prime-Time}

Caso 3: Televisión // Callejeros y 21 Días // 2005

Con la llegada del siglo XXI se instaura definitivamente la llamada tele-realidad, termino que empezará a aplicarse para definir talkshows o espacios relacionados con la "prensa del corazón", que de hecho acabarán substituyendo a los valores del melodrama, de los que eran herederas las populares tele-novelas. Esta realidad melodramática empezará también a convivir con nuevas formas de sensacionalismo mercantilista introducidas dentro de los géneros de investigación periodística, bajo etiquetas como "periodismo de inmersión". Desde su aparición, estos nuevos productos informativos o documentales prestan especial atención al concepto "droga", pretendiendo representar un modelo de reportaje preocupado por aspectos sociales, aunque pronto se hará evidente que sistemáticamente se recurre a la provocación sensacionalista impuesta por puros criterios económicos. El referente que certifica la aparición de este nuevo subgénero es el programa Callejeros (2005, Molinos de Papel), emitido por la cadena Cuatro.

Este programa pregona un modelo de periodismo con "compromiso social", en el que una pequeña cámara y un atrevido reportero se adentran en los barrios de grandes ciudades con el objetivo explícito de dar una versión "real" de la vida cotidiana a través del testimonio de sus vecinos. "La cámara se convierte en un personaje dramático, que quiere conocer historias y hurgar en los sentimientos de sus protagonistas. Su estilo de grabación imita a la vida misma, a través del plano secuencia, y consigue una gran identificación en los telespectadores, gracias al trato cercano de los reporteros" (Vázquez-La Hoz, 2012). Sin embargo, pronto descubriremos que ese "compromiso" vuelve a estar más ligado a los datos de audiencia que a la veracidad y el interés colectivo, así la impunidad y crudeza con la que se muestran situaciones relacionadas con el abuso de las drogas en nada contribuirán a un tratamiento del fenómeno que realmente ayude a evitar sus riesgos y reducir su mortalidad.

Pocos años después, esta misma cadena lanzará el referente "definitivo" de periodismo de inmersión: el programa 21 Días (2009, BocaBoca, Cuatro). A grandes rasgos, el formato propone que una periodista dedique tres semanas integras a realizar algún tipo de actividad controvertida - como dormir en la calle o someterse a una estricta dieta- bajo el pretexto de que, viviendo la situación en sus carnes, podrá ofrecer una mirada más profunda de la situación. Como no podía ser de otra manera, el fenómeno de las drogas no se pasó por alto por alto en esta nueva apuesta de tele-realidad. En su tercera emisión, el 27 de marzo de 2009, la reportera Samanta Villar emite su experiencia de estar "21 días fumando porros". El programa registró ese día su mayor dato de audiencia hasta el momento (18,3\% de share, 2.292 .000 espectadores), y en el global de sus cuatro temporadas solo sería superado por la emisión que le siguió, con lo que entendemos que esta entrega marca su punto álgido de popularidad.

\footnotetext{
${ }^{1}$ Este termino ha sido recientemente recuperado y emplazado en un contexto cultural inédito hasta el momento. Concretamente en una exposición titulada "Quinquis de los 80. Cine, prensa y calle", comisariada por Amanda Cuesta y Mery Cuesta, y exhibida entre 2009 y 2010 en el Centro de Cultura Contemporánea de Barcelona, en La Casa Encendida de Madrid y en Alhóndiga Bilbao.
} 


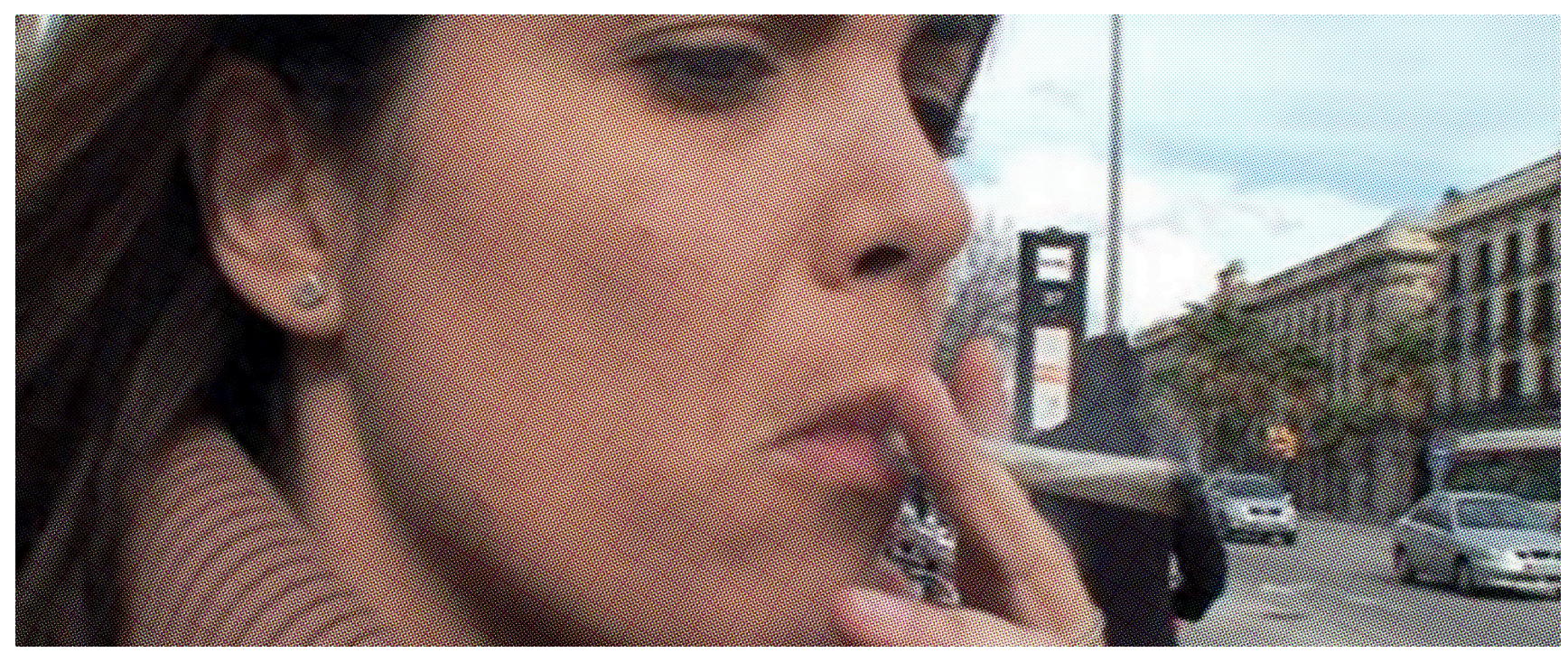

Sin embargo las críticas no se hicieron esperar, especialmente por parte de asociaciones canábicas y personalidades partidarias de la legalización, aunque también por parte de críticos de televisión que advertían una alarmante manipulación disfrazada de objetividad. La más contundente de estas críticas fue la redactada como carta a la dirección del programa por parte de Martín Barriuso, presidente de la Federación de Asociaciones Canábicas (FAC) y que, curiosamente, ha sido eliminada de la mayoría de portales de internet que en su día la publicaron. En ella afirmaba que "el programa de la Cuatro fue una muestra clásica de manipulación y propaganda antidrogas en el más rancio estilo. Se alteró el orden de los acontecimientos (el brutal colocón holandés, por ejemplo, se produjo varias semanas antes de rodar el resto) y se eliminaron varios días de material grabado (como la visita a la asociación MACA de Barcelona) para que todo encajara en el guión preestablecido. Se suprimieron los mensajes políticos de las asociaciones canábicas y se dio un peso exagerado a un pequeño grupo de ex-adictos que no representan ni de lejos la realidad de la mayoría de quienes fumamos porros." (Barriuso, 2009) Lo cierto es que partiendo de esta personalización de la experiencia, las conclusiones a las que se llegan son obviamente personales (valga la redundancia), basadas además en un consumo alarmantemente abusivo que poco o nada tiene que ver con la realidad cotidiana de la mayoría de consumidores, y que por tanto altera de facto esta realidad. Sin embargo, estas conclusiones se intentan universalizar y el programa termina con sentencias que parecen querer trascender el ámbito de la experiencia particular en el que se mueve el espacio, para convertirse en una especie de editorial o de moraleja.

\section{Una breve conclusión}

Con estos tres casos hemos pretendido mostrar una pequeña parte de lo que ha sido el método de investigación y de exposición de elementos realizado en la Tesis Doctoral anteriormente mencionada, y que se hará pública a mediados de este mismo año. El obligado trabajo de síntesis nos ha impedido mostrar otros ejemplos de producción audiovisual en los que el fenómeno de las drogas sí es tratado con veracidad, compromiso y un consecuente interés social. Sin embargo, nos vamos a permitir finalizar esta comunicación planteando una de las conclusiones generales alcanzadas al término de nuestra investigación, que obviamente ha sido más compleja y exhaustiva.

La construcción del imaginario colectivo de las drogas mediante los lenguajes audiovisuales, así como su modificación y posible evolución, está directamente vinculada con el contexto político bajo el que se desarrolla y gestiona el problema de la droga. En el caso concreto de España, este contexto se encuentra completamente condicionado por los "consensos" internacionales asumidos como Estado. No obstante, la inmensa capacidad de influencia que los lenguajes audiovisuales adquieren en la sociedad contemporánea les otorgan la incontenible capacidad de mostrar realidades incómodas, de proponer alternativas o de, simplemente, proporcionar información a la sociedad sobre aspectos de la droga que le son desconocidos. Sólo es necesario voluntad para hacerlo y determinación para romper con las imposiciones a las que obliga un discurso oficial y homogéneo sobre la drogas, que se encuentra cada vez más deteriorado y que exige nuevas formas de representación más cercanas a la realidad social. No obstante, esta sentencia constituye la definición de un punto de partida más que la de un objetivo alcanzado. Estableceremos aquí un punto y aparte en este proceso de investigación que nos ha permitido no sólo escrutar, sino también organizar, catalogar e interconectar centenares de manifestaciones fílmicas, televisivas y musicales, que han construido la historia de este país, en general, y en particular la de su forma de relacionarse, entender, sufrir y afrontar una controversia contemporánea internacional como el fenómeno de las drogas y sus problemas derivados. 


\section{FUENTES REFERENCIALES.}

BARRIUSO, M. (2009). "21 días de estupidez y porros" <http://www.f-enlace.org/index. php/actualidad/comunicados-informacionenlace/368-ante-reportaje-emiti- do-cuatro-21-dias--fumando-porros> [Consulta: 14 de septiembre 2014]

BOCABOCA. (2009). 21 días fumando porros. Madrid: Cuatro. (Programa de televisión)

DE LA LOMA, J.A. (1977). Perros Callejeros. Madrid: Films Zodiaco/Profilmes. (Película)

ESCOHOTADO, A. (1989). Historia general de las drogas. Madrid: Espasa.

MEGÍAS, E.(dir.) et al. (2004). La percepción social de los problemas de drogas en España. Madrid: FAD Fundación de Ayuda contra la Drogadicción.

MOLINOS DE PAPEL, MEDIAPRO. (2005). Callejeros. Madrid: Cuatro. (Programa de televisión)

ROMANÍ, O. (1999). Las drogas: Sueños y razones. Barcelona: Ariel.

TRUCHADO, J. (1977). Juventud Drogada. Madrid: Amanecer Films. (Película) 